Reprod. Nutr. Dévelop., 1986, 26 (1 B), 265-266.

\title{
Comportement alimentaire d'un troupeau caprin dans un taillis de chêne vert
}

M. MEURET, Elisabeth LÉCRIVAIN, Bernadette LECLERC

Unité d'Ecodéveloppement, I.N.R.A. 84140 Montfavet, France.

Summary. By studying the grazing behaviour of a flock of goats in an holm-oak copse, data were obtained on the intake rate and the botanical composition of the diet.

Cette étude concerne un troupeau caprin pâturant en très forte charge instantanée (100 à 200 animaux/ha) des parcs clôturés dans un taillis dense de Chêne vert (Quercus ilex), non exploité depuis 40 ans, dans le but de réaliser un débroussaillage. Dans ce cadre, nous avons été amenés à quantifier la ration ingérée par différentes méthodes : enregistrements zootechniques, mesures phytoécologiques et observation directe du comportement alimentaire. Les principaux résultats obtenus à l'aide de cette dernière méthode sont présentés ici.

Matériel et méthodes. Le troupeau est constitué de 150 chèvres de la race du Rove et de la race dite commune, d'un poids moyen de $45 \mathrm{~kg}$. L'alimentation provient pour moitié, en moyenne, du taillis de chêne ; le reste est fourni par une luzerne pâturée quotidiennement. Sept parcs clôturés dans le taillis, d'une superficie de 0,5 à 1,5 ha, sont utilisés en rotation sur six mois à raison de 2 à 9 jours par passage. La durée quotidienne de présence en parc varie de 4 à $6 \mathrm{~h}$, le matin ou l'après-midi, en fonction des conditions climatiques.

La méthode utilisée pour l'essai de quantification de l'ingestion en parc est I'observation directe de la prise alimentaire (Allden et Whittaker, 1970; Chacon et Stobbs, 1976), adaptée pour la circonstance. Deux observateurs sont présents simultanément, de l'entrée à la sortie du parc, avec une fréquence moyenne d'un jour de relevé sur deux jours d'utilisation du parc. Le premier relève par observation directe durant $20 \mathrm{~min}$, à intervalle de $30 \mathrm{~min}$, l'activité d'un échantillon de 50 individus marqués. Les proportions d'animaux dans chacune des activités (pâturage, déplacement, repos) permettent de calculer la durée totale moyenne de pâturage. Le second suit successivement des animaux tirés au sort entre chaque relevé. Il compte durant $10 \mathrm{~min}$, à intervalle de $20 \mathrm{~min}$, les coups de dents (C.D.) portés sur les différentes espèces végétales. II mesure ainsi la fréquence des coups de dents (C.D./min). Par ailleurs, il évalue le poids du coup de dents (g M.S./C.D.) par présentation à bout de bras, au sein du taillis, de rameaux pesés en frais (Meuret et al., 1985), ou par simulation manuelle.

Les résultats présentés ne concernent qu'un seul parc 10,83 ha 180 animaux/ha), utilisé à trois reprises : du 17 au 25/4, du 5 au $8 / 7$ et du 29 au $31 / 8 / 1984$.

Résultats et discussion. Lors du premier passage en parc, le taux horaire de pâturage a été très élevé dès le premier jour et il est resté relativement constant ( $87 \pm 4 \%$ : voir fig. 1). L'éleveur n'ayant augmenté que de $8 \mathrm{~min} /$ jour en moyenne la durée de présence en parc, l'augmentation quotidienne de la durée de pâturage a été faible. La forte diminution du poids du coup de dents à partir du $4^{e}$ jour $n^{\prime}$ a pas entraîné une augmentation de la fréquence des C.D., et dès lors, la vitesse d'ingestion (C.D./min) a régulièrement diminué. La multiplication des trois variables (poids du C.D., fréquence des C.D. et durée de pâturage) permet d'esti- 
mer la quantité de matière sèche ingérée quotidiennement. L'ingestion a diminué, non seulement au cours de chaque passage, mais aussi d'un passage à l'autre dans un même parc, cela en raison de l'ajustement de l'apport de luzerne à la diminution de la disponibilité en parc.

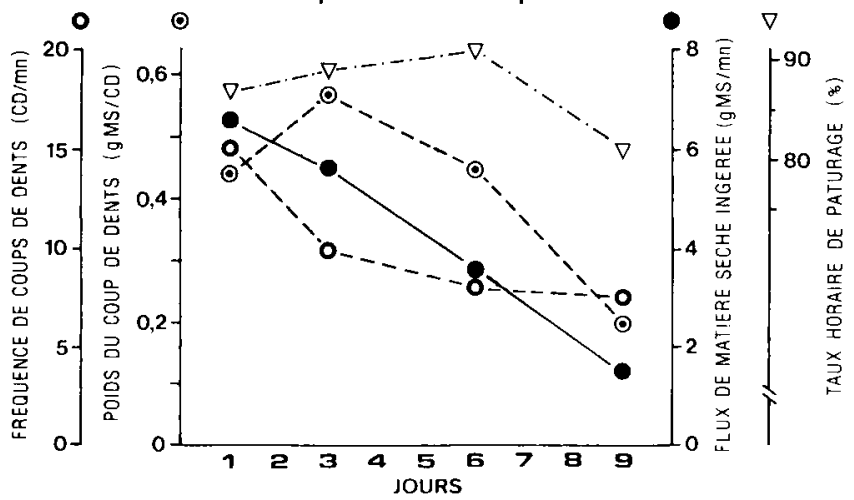

FIG. 1. - Evolution des principales composantes du comportement alimentaire (premier passage en parc : 9 jours en avril).

Cette dernière a influencé de façon prépondérante l'évolution de la composition botanique de la ration (fig. 2). On note, à chacun des trois passages, l'importance constante du Chêne vert, et le fait que les animaux ont progressivement adapté leur hauteur de broutage à la diminution de la disponibilité de cette espèce : jusqu'à $1,4 \mathrm{~m}$ en avril, $1,8 \mathrm{~m}$ en juillet et $2 \mathrm{~m}$ en août. Cet ajustement ne semble pas suffisant, car on voit se substituer au Chêne des espèces non consommées habituellement, comme le Fragon (Ruscus aculeatus), qui atteint $14 \%$ M.S. de la ration à la fin août. Les espèces herbacées sont très peu représentées (moins de $5 \%$ M.S.), d'une part car elles sont peu abondantes dans le parc (12\% M.S. de la phytomasse disponible), mais également car elles ne sont pas préférées aux espèces arbustives et aux lianes.

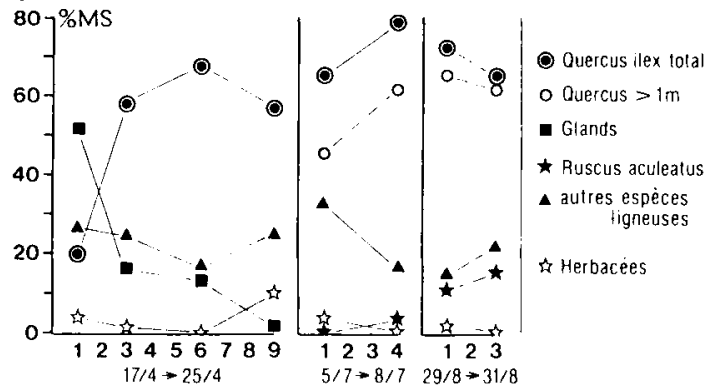

FIG. 2. - Composition botanique de la ration ingérée (\% M.S.) durant les trois passages dans le parc.

En conclusion, bien que la méthode soit imparfaite (en particulier, la détermination du poids du coup de dents reste encore trop incertaine), l'observation directe permet d'appréhender dans queiles limites et avec quelle dynamique l'animal peut ajuster son comportement alimentaire à une diminution rapide des ressources pour couvrir ses besoins tout en assurant un débroussaillage.

Allden W. G., Whittaker I. A., 1970. The determination of herbage intake by grazing sheep The interrelationship of factors influencing herbage intake and availability. Aust. J. agric. Res., 21, 755-766.

Chacon E., Stobbs T. H., 1976. Influence of progressive defoliation of a grass sward on the eating behavior of cattle. Aust. J. agric. Res., 27, 709-727.

Meuret M., Bartiaux-Thill N., Bourbouze A., 1985. Evaluation de la consommation d'un troupeau de chèvres laitières sur parcours forestier : Méthode d'observation directe des coups de dents, méthode du marqueur oxyde de chrome. Ann. Zootech., 34, 159-180. 